\title{
Blockchain Technology Implementation for Medical Data Management in Malaysia: Potential, Need and Challenges
}

\author{
Fariha Anjum Hira ${ }^{1}$, Haliyana Khalid ${ }^{1}$, Siti Zaleha Abdul Rasid ${ }^{1,2}$, \\ Shathees Baskaran ${ }^{3}$, Alam Md Moshiul ${ }^{4}$ \\ ${ }^{1}$ Azman Hashim International Business School, Universiti Teknologi Malaysia, \\ Kuala Lumpur 54100, Malaysia \\ ${ }^{2}$ University of Business and Technology, Jeddah, Saudi Arabia \\ ${ }^{3}$ Azman Hashim International Business School, Universiti Teknologi Malaysia Skudai, Johor, Malaysia \\ ${ }^{4}$ Razak Faculty of Technology and Informatics, Universiti Teknologi Malaysia, \\ Kuala Lumpur 54100, Malaysia
}

\begin{abstract}
This study highlights several challenges along with the potential and need for blockchain technology implementation in the Malaysian healthcare industry context. The systematic review unearths potential technological, organizational, environmental, individual-level acceptance concerns and challenges associated with deploying blockchain to support electronic health records. As such, this research serves as the starting point for a chain of studies aiming at detecting, analyzing, and responding to such demands. The study is expected to guide policy and decision-making procedure of a secure and resilient health information exchange for healthcare stakeholders of developing nations such as Malaysia.
\end{abstract}

Keywords - blockchain, EHR, health information exchange, lifetime health record.

DOI: 10.18421/TEM111-08

https://doi.org/10.18421/TEM111-08

Corresponding author: Fariha Anjum Hira,

Azman Hashim International Business School, Universiti Teknologi Malaysia, Jalan Sultan Yahya Petra, 54100, Kuala Lumpur, Malaysia.

Email: hirafa92@gmail.com

Received: 22 September 2021.

Revised: 12 January 2022.

Accepted: 17 January 2022.

Published: 28 February 2022.

(cc) BY-NC-ND (C) 2022 Fariha Anjum Hira et al; published by UIKTEN. This work is licensed under the Creative Commons Attribution-NonCommercial-NoDerivs 4.0 License.

The article is published with Open Access at www.temjournal.com

\section{Introduction}

This Electronic medical record (EMR) was introduced in the 1960s. Later, since the 1980s, manual medical records began to be replaced by EMR to fully digitalize (paperless) the medical data management system [1]. Unfortunately, the healthcare entities continue to maintain paper-based records for a certain period along with EMRs; thus, medical record-keeping has become hybrid (both paper and digital) [2]. The healthcare ecosystem is not fully digitalized yet. EMR is an internal system; when shared with other departments, it becomes part of Electronic Health Record (EHR), a health facilitybased inter-organizational exchangeable system used by health professionals [3]. However, the use of EHR remains controversial. Many researchers claim that EHRs are mainly used for administrative purposes, such as financial transactions and invoicing [4]. As mentioned by Leeming, Cunningham \& Ainsworth [5], "EHRs were never designed to manage the complexities of multi-institutional, lifetime medical records. As patients move between providers, their data becomes scattered across different organizations, losing easy access to records. As providers, the patients are not the primary stewards of EHRs, they face significant hurdles in viewing their reports, correcting erroneous data, and distributing the information". The core potential of EHR is improving patient safety through digitalized medical data management and exchange. The prospect of EHR is not adequately utilized. Therefore, although the traditional EHRs have existed for decades, very few health entities are fully digitalized.

The number of patients is increasing with the increase in the global population. Considering the voluminous medical data, the existing EHRs have 
become insufficient to fulfill patients' demands and control their health information. Undoubtedly, the current medical data management process has various practical problems. For instance, in 2005, during Hurricane Katrina in New Orleans, numerous paper-based medical records were destroyed. The loss of medical records leaves patients vulnerable to medical errors given healthcare professionals' failure to link the patients' present health conditions and their medical history considering diagnosis, drugs, effects, assessment of surgery risks, etc. Thus, the requirement for complete digitalization of medical data became apparent.

Implementing EHR to digitalize the healthcare ecosystem is considered necessary for patient safety. Digitalization would minimize medical error, enable efficient medical data exchange, ensure data security, and protect patients' privacy. EHR has some limitations and pitfalls that need to be fixed. The issues include: (i) difficulty managing voluminous data, (ii) risk of data mishandling, linkage, data breach because of third party involvement in data administration, (iii) unavailability of patients' medical history at the point of care due to centralized data management [6]. Accepting that EHR has limitations, healthcare researchers and industry players are continuously searching for tools or mechanisms that can support EHR to overcome those drawbacks.

In 2008, blockchain technology (BcT) was introduced by Satoshi Nakamoto. It is a "peer-to-peer electronic cash system," the underlying mechanism of bitcoin cryptocurrency transactions [7]. BcT is a combination of inputs obtained from three computer networking fields, namely decentralized networking, the process of consensus, and encryption. In that sense, it is not an innovation but a combination of existing features in a new-fangled form. BcT can enhance confidentiality, privacy, and security of the data [8].

Consequently, it has emerged as a powerful disruptive innovative technology. Industries such as banking, social services, risk management, and supply chain management are already implementing $\mathrm{BcT}$ for their business operation. BcT seems to be the appropriate mechanism to tackle issues associated with traditional EHRs. In 2016, the technology started to gain interest from healthcare researchers [7].

Unlike other countries, the Malaysian healthcare industry is highly regulated and unique in-service delivery nature. Therefore, the industry needs a particular focus on state-of-the-art technology adoption patterns to identify the possible implications of BcT. Also, unearthing the factors that can hinder $\mathrm{BcT}$ intervention is required to develop an appropriate regulatory framework, policy, implementation. Health technology adoption in Malaysia has traditionally shown slow progress. The study on BcT in the Malaysian healthcare industry context is highly lacking. There are very few studies on BcT conducted in Malaysia. The studies mainly focus on halal supply chains, identity management, transport industry, crowdfunding, banking, cryptocurrency, supply chain management, value chain management, and smart cities. In short, BcT in Malaysia is still in its infancy stage. To the best of our knowledge, none of the studies have been on blockchain EHR. There is a lack of understanding in this regard which is the motivation of this study. This study seeks to answer the questions, which medical data management issues can be solved using BcT? Why is BcT necessary for the Malaysian healthcare industry? and what challenges may hinder the BcT adoption in Malaysia's healthcare ecosystem?" This systematic review aims multi-fold; (i) to explore the potential of blockchain-based medical data management over traditional EHR-based mechanisms, ii) to understand the necessity of BcT in the Malaysian healthcare industry, iii) to identify the possible challenges associated with BcT implementation in the country.

\section{Method}

This study uses the existing literature for analysis. Systematic literature review (SLR) is a widely used method of scientific research mining and research in a particular area of interest. This approach is distinguished by the implementation of explicit protocols and conditions that can theoretically mitigate bias. SLR was conducted to respond to the present study's research questions and cover relevant literature on the central search of BcT. A keywordbased literature search is on scholarly databases to review and discuss the findings pertinent to this study between 2009 and 2021. Only English-language articles have been included. The keywords "blockchain technology" and "healthcare" have been used with search terms in various forms, including "Malaysia," "Information Exchange," "data breach," "MyHIX," "advantages," "digital health," etc. The first skimming phase was conducted by looking at the title, abstract, and keywords relevant to this study. Then the full texts were perused to check for relevance, and a total of 153 articles were retained for further analysis. Figure 1. shows the step-by-step process of data search and screening. 


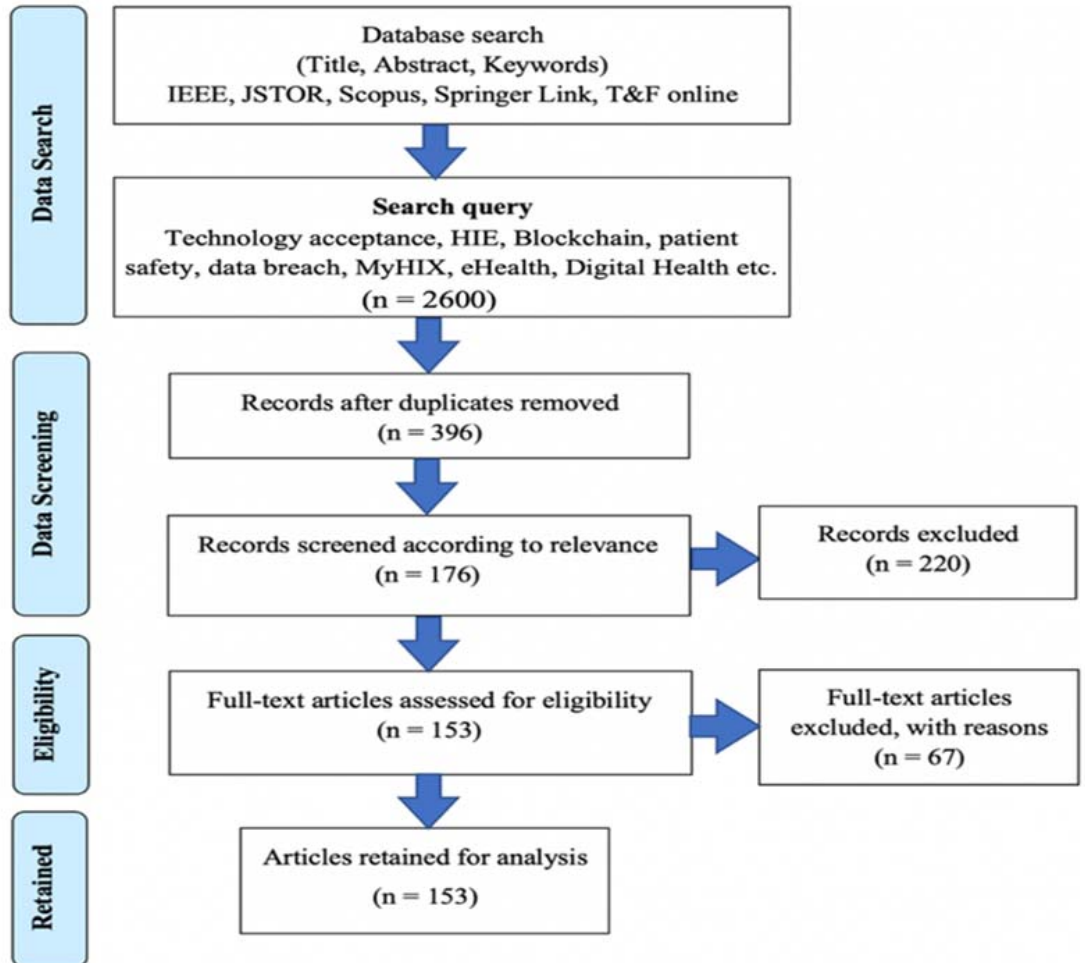

Figure 1. PRISMA flow diagram of data search

The papers mainly discussed the technological $(70 \%)$ aspect of BcT, followed by the organizational perspective (18\%). Less attention was given to the Environmental factors (10\%) aspect of $\mathrm{BcT}$ in the healthcare industry context.

\section{Related Work}

This section highlights the related studies that highlighted issues associated with $\mathrm{BcT}$ adoption from a developing country perspective as Malaysia is a developing country.

With a focus on India as a developing country, Sharma and Joshi [9] present 15 barriers to BcT implication, including technological issues such as lack of data storage capability, standardization, scalability, and high initial and maintenance costs. The study further highlights that lack of research on technology adoption is one of the main reasons to hinder the diffusion of BcT, particularly for the developing countries' healthcare industry. Furthermore, lack of trust, knowledge, and understanding of the technology, data privacy concerns, risk associated with medical data may hinder the individual level adoption of BcT. At the organizational level, lack of management support, infrastructure insufficiency, shortage of expertise, poor project management are the issues that require attention. Also, legal restrictions regarding medical data management need to be revised as currently, it is not blockchain-friendly. Studies on BcT implementation in the Healthcare Industry predominantly focused on empirical evidence for
BcT use. Like the study by Sharma and Joshi, 13 critical success factors were highlighted.

Angraal, Krumholz, and Schulz [10] mentioned the use case of BcT-enabled EHR (i.e., Guardtime, MedRec) that are successfully running for exchanging medical data, which supports that the BcT is applicable for medical data management. Also, BcT has potential application in medical supply chain management. However, privacy, regulatory concerns, technical issues such as data storage and distribution may hinder the progress of BcT adoption in the healthcare industry. Moreover, the paper heavily focused on the technical limitations of the BcT.

A systematic literature review by Tandon et al. [11] highlights the lack of review studies on BcT adoption in the healthcare context attempting to unearth the barriers and potential. The study identifies that medical data management can facilitate the use of $\mathrm{BcT}$ as it provides patients with control and validation of their medical data. Removal of intermediaries also depresses the risk of a medical data breach. Also, the lack of research on understanding users' perspectives is alarming because BcT is a disruptive technology. Thus, organizational, individual, technological, and environmental (i.e., regulatory, legal) changes are necessary. However, the bibliometric study highlights several limitations of current research and mentions some potential barriers that may hinder BcT adoption in the healthcare industry but suggest predominately to research architecture optimization, legal compliance, technology integration, and contribution of $\mathrm{BcT}$ for 
the supply chain. Furthermore, BcT adoption issues are not discussed in detail, only that there is a need to study these issues in the future.

Durneva, Cousins, and Chen [12] highlight that BcT for HIT ensures privacy and security of medical data and aids interoperability of heterogeneous HITs, thus improving health care quality. Also, use barriers include security and privacy concerns, user resistance, lack of expertise, technology, implementation costs, and technological issues (i.e., inefficient consensus algorithms, BcT integration with existing HIT). However, user resistance issues have not been discussed in detail. Although the study shows a breakdown of EMR/EHR architecture-based research $(51 \%$, ) data security, privacy-oriented research $(53 \%)$, it did not specify the status of BcT adoption research.

Prior studies attempted to unearth barriers and possibilities of BcT in the healthcare industry and developing country context. However, none of the studies evaluated the unique setting of the Malaysian healthcare industry. The present study, therefore, attempts to fill these knowledge gaps by exclusively focusing on Malaysia. Currently, there is a lack of literature on health information technology (including blockchain EHR) in the hospital setting. This is one of the first reviews on Blockchain EHR in Malaysia, particularly to researchers' best knowledge. Therefore, this study attempts to shed light on blockchain EHR implementation need and potential berries from the related studies considering developing country context and prior health information technology studies conducted in the
Malaysian healthcare industry context. The available studies on health information technologies in Malaysia are older and explicitly public healthcare facility focused. Thus, the proposition of this systematic review is limited to the need and potential barriers of blockchain EHR implementation considering Malaysian public healthcare facilities.

\section{Blockchain for Medical Data Management in Malaysia}

\subsection{Potential of Blockchain}

Blockchain is a distributed ledger technology. If used as an underlying mechanism of EHR (blockchain EHR), the medical data exchange becomes tamper-proof, secure, and scalable. Also, the distributed nature of $\mathrm{BcT}$ can remove third-party involvement, data silos. It establishes trustless medical data exchange among authorized parties. BcT noticeably increases the efficiency of EHR compared to traditional EHR, thus, improves coordination of care.

Furthermore, healthcare cost reduction is possible through better insurance claim coordination, treatment, and diagnosis redundancy avoidance. The data auditing makes the record immutable, and validation ensures authentication of the medical data [13]. The healthcare industry's medical data management issues that $\mathrm{BcT}$ can address have been listed in Table 1., considering the Malaysian healthcare industry context.

Table 1. Potential of blockchain in addressing medical data management and exchange issues

\begin{tabular}{|c|c|c|c|}
\hline Issues & Explanation & Current challenge & Blockchain implication \\
\hline $\begin{array}{l}\text { Identity } \\
\text { authentication }\end{array}$ & $\begin{array}{l}\text { Information to verify that } \\
\text { someone is who she claims to } \\
\text { be. Example: Username, } \\
\text { password, or thumbprint. }\end{array}$ & $\begin{array}{l}\text { Identity management of health } \\
\text { entities is password-based, } \\
\text { involving shared secrets } \\
\text { exchanged and stored in an } \\
\text { insecure system. }\end{array}$ & $\begin{array}{l}\text { BcT enabled Identity } \\
\text { authentication provides only } \\
\text { the patent with a private key; } \\
\text { stakeholders involved in a } \\
\text { transaction must sign in } \\
\text { using a correct private key. }\end{array}$ \\
\hline $\begin{array}{l}\text { Information } \\
\text { describing the } \\
\text { information }\end{array}$ & $\begin{array}{l}\text { Various data among } \\
\text { stakeholders and records of } \\
\text { data transactions. Details on } \\
\text { patients' expectations about } \\
\text { the way of their data use, } \\
\text { consent management. }\end{array}$ & $\begin{array}{l}\text { There are no audit trails of who } \\
\text { accessed patients' data. Some } \\
\text { hospitals still rely on paper-based } \\
\text { medical records. }\end{array}$ & $\begin{array}{l}\text { The presence of an audit trail } \\
\text { means that there is the } \\
\text { complete documentation of } \\
\text { events related to the creation, } \\
\text { modification, and delectation } \\
\text { of electronic records. }\end{array}$ \\
\hline $\begin{array}{l}\text { Actions that } \\
\text { various } \\
\text { participants } \\
\text { are authorized } \\
\text { to perform }\end{array}$ & $\begin{array}{l}\text { An access policy specifies the } \\
\text { access rights and privileges of } \\
\text { each participant. For example, } \\
\text { insurance companies cannot } \\
\text { have access to patients' } \\
\text { confidential medical records. }\end{array}$ & $\begin{array}{l}\text { Various parties are authorized to } \\
\text { act based on patients' data. } \\
\text { Patients have no control over their } \\
\text { medical data. }\end{array}$ & $\begin{array}{l}\text { BcT prevents unauthorized } \\
\text { and illegal assess to data. } \\
\text { Patients hold ownership and } \\
\text { ultimate control over their } \\
\text { health information. }\end{array}$ \\
\hline Efficiency & $\begin{array}{l}\text { The inefficiency of } \\
\text { administrative, logistic, and } \\
\text { service delivery processes } \\
\text { leads to higher costs and } \\
\text { consumers' time a lot but }\end{array}$ & $\begin{array}{l}\text { Inefficient procedures to transfer } \\
\text { data across healthcare service } \\
\text { providers. Policy and regulatory } \\
\text { heterogenicity across } \\
\text { jurisdictions. }\end{array}$ & $\begin{array}{l}\text { A patent can access their up- } \\
\text { to-date health information } \\
\text { and forward them to } \\
\text { caregivers or other parts as } \\
\text { required. }\end{array}$ \\
\hline
\end{tabular}




$\begin{array}{ll}\begin{array}{l}\text { Information } \\ \text { exchange } \\ \text { difficulty }\end{array} & \begin{array}{l}\text { Medical data should be easily } \\ \text { exchangeable among various } \\ \text { stakeholders as per necessity. }\end{array} \\ & \begin{array}{l}\text { HIE standard for the entire } \\ \text { healthcare ecosystem is a must } \\ \text { interpretability } \\ \text { for flowless data exchange } \\ \text { among caregivers. }\end{array}\end{array}$

$\begin{array}{ll}\text { Data } & \begin{array}{l}\text { Medical history should be } \\ \text { radiality available at the point } \\ \text { of care for the physician's firm } \\ \text { decision-making. }\end{array}\end{array}$

Several health entities that a patient approaches for $\begin{array}{ll}\text { Scattered } & \text { patient approaches for } \\ \text { patient history } & \text { treatment purposes hold }\end{array}$

Inconvenience secure sharing different medical information pieces of a particular patient. Sharing health information is a time-consuming and complex process. In Malaysia, sometimes medical data sharing occurs through email, WhatsApp.
The EMR only records data for departmental use, and EHR allows inter-departmental use of the data.

\section{Different entities have their} standard. From a business view, health entities are competitors. Therefore, there is fear of others getting a competitive advantage which restricts health entities from data sharing.

Patients cannot hold a copy of their treatment history; therefore, they cannot ask for a second opinion. They are also unable to provide it to the caregiver in case of an emergency. Currently, data retrieval involves a third-party and time-consuming process.

A patient's single lifetime health records are scattered and nearly impossible to gather under the current medical data management mechanism.

Patients need to request formally to the hospital for their medical data. The process takes time, costs money still sometimes, patients don't get the information.
A lifetime health record (LHR) can be created, readily available to the caregiver with the patient's authorization.

BcT can tie up all healthcare entities under a single datasharing mechanism maintaining the same standard. This will be helpful for caregivers in therapeutic decision-making for patients. The patients can have a hold over their medical records. Therefore, medical data can be accessed at the point of care. BcT elementals the need for third-party involvement, thus reducing the time for data accessibility.

\section{Blockchain EHR can facilitate "1 person, 1 record" formation. It can remove data silo.}

BcT can convert the system as patient centric. The patients will be the decisionmakers while sharing their medical data.

Source: several sources including [14], [15]

The Malaysian government also recognizes the benefits of the BcT as the Ministries, including healthcare organizations, are focused on utilizing it in upgrading countries' industries to Industry Revolution 4.0 (IR4.0) level. Malaysia and Singapore agreed to work collaboratively on vaccine certification. The blockchain-based app "The Immunity Health Passport" has been accepted by Singapore for COVID vaccination information exchange for Malaysian travelers to Singapore. This is Malaysia's first initiative to exchange health information using a BcT-based application. The BcT is not hype because there are successful use cases such as Deloitte, Accenture, Guardtime for storing, managing, and exchanging medical data [16]. For instance, Estonia initiated partnering with Guardtime in 2011 and, since 2016, began using blockchain EHR. Blockchain EHR provides tamper-proof, secure, scalable medical data exchange. It eliminates data silos, thereby improving EHR efficiency. Besides, the $\mathrm{BcT}$ can reduce the cost of care and be convenient for insurance claims for patients. Furthermore, healthcare researchers get anonymous data from blockchain EHR, thus costing data collection offsets [13].

\subsection{Necessity of Blockchain}

The need for BcT for the Malaysian healthcare industry is in line with its benefits, such as safeguarding from medical error, data safety, patient privacy, and patient ownership over medical data. Blockchain as an underlying mechanism of EHR establishes distributed ledger where all information is audited and validated. There is no need for intermediaries' involvement; thus, the risk of a data breach is minimal. Due to the unique features like this chain of blocks holding patients' information can be developed with the help of BcT, the LHR of a patient can be generated from blockchain EHR. Furthermore, BcT based EHR is likely to have improved efficiency; thus, it indirectly assists in establishing nationwide health information exchange (HIE).

Medical error is a prime cause of mistreatment; thus, posing a direct threat to patient safety. Medical data availability is essential for evidence-based medicine. Treatment and diagnosis are not always decided based on scientific evidence. Treatment choice varies from physician to physician, and patients get different physicians' instructions for the 
same problem. Around $90 \%$ of treatment decisions depend on prior treatment, whereby information plays an imperative role in the healthcare industry to support quality care delivery. As a result, patients' previous health record availability is vital for quality care [16]. In Malaysia, the medical error for prescribing stands at $8.8 \%$ [17], administration at $8 \%$, and dispensing at $1.6 \%$, respectively [7]. The BcT eliminates data sharing barriers, which can reduce medical errors and improve patient safety.

The expression "Every blessing has a curse" also applies to the utilization of health information technologies. Although the EMRs/EHRs are efficient data management tools, the intermediaries involved in centralized systems pose data breach risks. Medical data contains confidential and sensitive information of patients that should be safely stored and cautiously shared due to security risks of system failure [18], mining attack, storage attack, and dropping episode [19]. Therefore, the privacy and security of medical data are a worldwide concern.

Current EMR/EHR is not capable of providing sufficient privacy and security for medical data. In 2015, the medical data breach record exceeded $112,000,000$. A medical data breach could threaten patients' personal and social status, even life. In Malaysia, data breach incidents have occurred in the recent past. A major data leak of over 46 million smartphone users took place in 2017. In 2018, around 220,000 Malaysian organ donors and their family members' personal information leaked online [20]. Elimination of intermediaries will be more beneficial than the point-to-point information-sharing mechanism [21], which can be achieved by enabling the $\mathrm{BcT}$ for medical data sharing.
Implementing BcT enabled HIE to enable patients to have direct control over their health records. The transaction process of the $\mathrm{BcT}$ is transparent. It allows transacting parties to trade digitally embodied properties without intermediaries in an immediate and immutable peer-to-peer framework. Figure 2. demonstrates the steps involved in a health information transaction between stakeholders. The process begins with the data transaction proposal. The cryptographic signature is added, and the system broadcasts the data among multiple stakeholders involved for validation. Once validated, the data attached to the block occurs; thus, the transaction finishes. Therefore, the patient is the authorizing person to permit and validate the transaction.

Furthermore, a patient can decide on to whom s/he wants to share the information. The mechanism eliminates the need for third-party involvement, provides data ownership authority to the patient, and is immutable. Data cannot be manipulated; the patient can decide who s/he needs to share which data; caregivers of any health entities can access data as per patients' needs. EMR, the EHR-based medical data management, has a few limitations, such as security of medical data, data ownership, and integrity [8]. The concept of medical data management and data ownership has changed over time from Healthcare 1.0 to Healthcare 4.0, as illustrated in Figure 3. [22]. In the 1960s, under Healthcare 2.0, electronic medical record-keeping was introduced [1], which was later replaced by electronic records or e-record and eventually by the EMR [2]. The e-record has been known as EMR since the Healthcare 3.0 era.

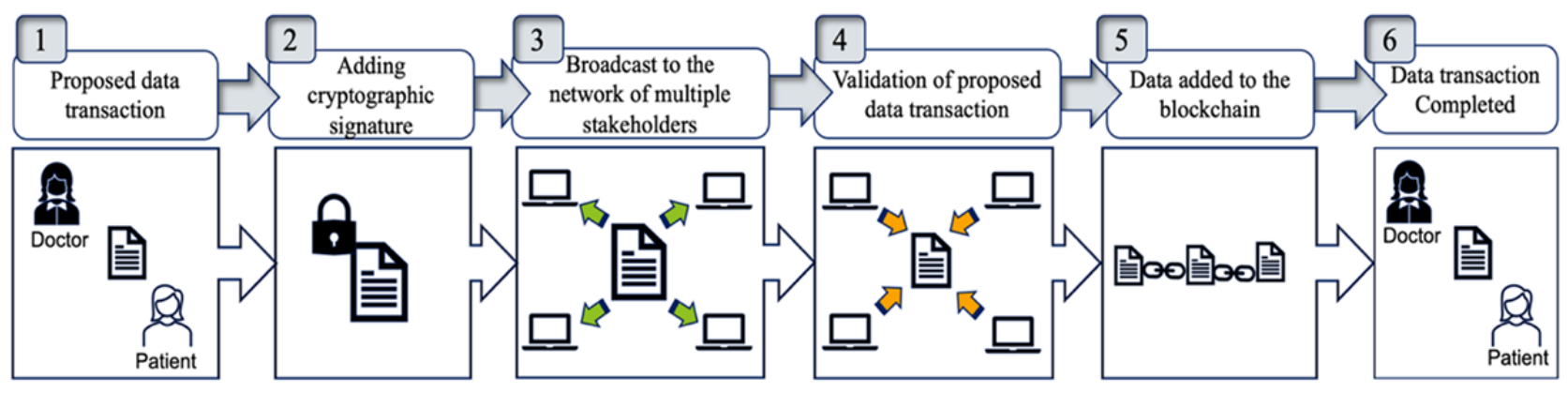

Figure 2. Secured medical data exchange steps in blockchain-based data exchange
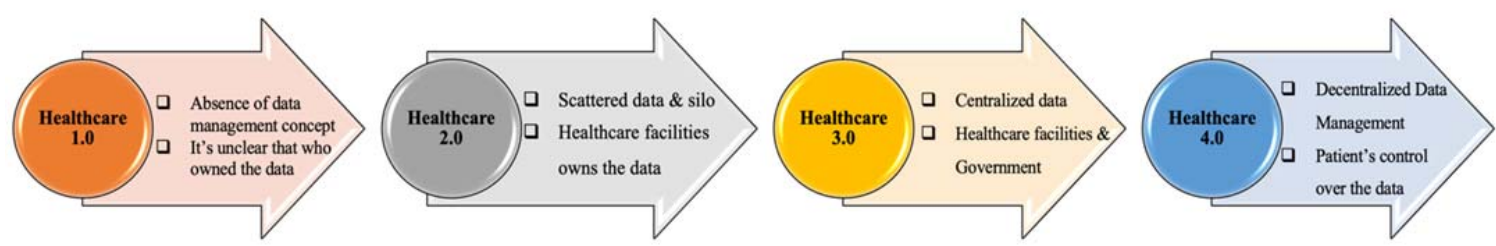

Figure 3. Change in medical data ownership over the period 
In Malaysia, EHR data is available to authorized personnel and for physicians' reference only. Even the patients have little to no influence or keep their medical data. It seems that the EHR serves the healthcare facility and not the patients. Instead of being patient-centric, the system became more physician-centric [23], [24]. This is where the Malaysian healthcare industry is standing now. In Blockchain EHR, patients cannot alter their medical data but can access and decide to whom the information is to be shared [24], [25].

Malaysia health information exchange is known as MyHIX [26], [27]. In use since November 2008, it is an EHR integration engine for exchanging health information in the form of discharge summaries among connected healthcare facilities via an online virtual private network. Nationwide MyHIX coverage will allow caregivers to access patients' medical records. It will improve the quality of care. Also, the LHR will continuously get updated in public healthcare facilities. Malaysian government targets to achieve nationwide MyHIX coverage. In line with this target, the EMR implementation and development of LHR are not yet established. The government, therefore, has undertaken EMR implementation of the project to include all 145 public hospitals. However, to date, only 10 hospitals and 1 clinic have been integrated. One of the primary reasons behind the low number of healthcare facility integration under MtHIX is the inefficiency of traditional EHR. Since blockchain EHR can improve efficiency, it can support the establishment of nationwide MyHIX network coverage.

The concept of lifetime health record (LHR) has been derived from the idea of representing a patient's life-long clinical history at the point of care with reports from various facilities of healthcare settings where $\mathrm{s} / \mathrm{he}$ has received medical care. An LHR contains the patient's medical information, including medication, past treatment, illness history. High-risk patients, such as cancer and asthma patients, can be identified without further action if the LHR is available. It is equally essential during infectious disease outbreaks such as COVID-19. During such a public health emergency, a well-positioned distributed health information exchange (HIE) mechanism can benefit from decision-making on treatment procedures.

In healthcare facilities with blockchain EHR, patients' information during a visit to any of them gets added as a block to the blockchain. The Ministry of Health, Malaysia, wants to have one patient one record system for every individual. Unfortunately, the project was halted in 1997 [28]. The BcT can improve efficiency and remove the pitfalls of the current EHR. To fulfill that target, BcT is needed.

\subsection{Challenges of Blockchain Implementation}

Technology adoption in the Malaysian healthcare industry is traditionally slow [27]. The BcT implementation is likely to encounter several issues, including user resistance. This article assesses BcT's potential to connect the health industry stakeholders and entities via information technology. From developing country and Malaysia focused information system (IS) literature, four challenges have been identified: technological, organizational, environmental, and human-related/individual level challenges.

\subsubsection{Technological Challenges}

The first technical barrier of BcT implementation is the technology itself due to its immaturity. Overcoming initial technical obstacles is essential for the success of technology implementation. The technical skills must first resolve technological barriers that the technology might have created. The technological challenges include compatibility in information exchange and transaction, time in processing transaction, block size, distributed ledger system, cybercrime, newness, standard infrastructure development, component standardization, and technological capacity building.

However, ensuring the availability of these technical capabilities is a significant obstacle in an innovative technology implementation process [23]. This applies to BcT initiatives because organizations (i.e., health entities) are beginning to implement the $\mathrm{BcT}$ for decentralized infrastructure. The unrivaled nature of the technology, lack of information, documentation, and the sheer number of BcT platforms aggravate the challenges even more. Another obstacle of BcT implication may occur due to the capability of the existing IT architecture of health entities.

Consistency of the current HIS and harmonization of hardware and software between participating entities is critical. For instance, the EHR of hospital A and Hospital B may not be compatible with each other. Thus, an intricate problem of system integration and assimilation may occur. There is an extreme shortage of lack of interpretability due to the newness of the technology. The missing of an industrywide standard system hinders the capability of inter-organizational exchange of data [29].

A standard for the BcT has not been set in the Malaysian healthcare industry context, which needs to be introduced. The BcT-specific extension is required to tackle technical issues of the technology implementation. Data interpretability is possible only if the data can be interpreted in the same standard manner. Currently, the EMR, EHR data produced by 
a department in many cases varies from other departments, and it is challenging to analyze and interpret the data's meaning. BcT implementation requires significant policy reform, which can be difficult, time-consuming, and highly challenging to achieve.

\subsubsection{Organizational Challenges}

Organizations need to invest substantially at the initial stage, which raises the question of return on investment, participation cost, lock-in effect, and parallel IT maintenance, which involves financial risk. Therefore, organizational readiness, innovativeness, and risk-taking capability should be present. Organizations may be reluctant to implement BcT due to the sizable initial investment and its indefinite benefit. BcT brings along huge change requirements to an organization, and project management issues are likely to occur. Therefore, with top management support, other challenges associated with organizational cultures, such as awareness of the need for change, the benefit of such change must be positively nurtured within the corporate cultural ecosystem. For instance, employees' involvement in project management from the very beginning could develop an understanding of the need for accepting BcT for HIE. The administration must also play their role in assuring appropriate training, supporting facilities, and dealing with employees' fear of job security to reduce change resistance, particularly among physicians. In short, sound organizational change management is required.

Moreover, organizational behavior and development intervention and human resource management will be impacted by the BcT implementation for HIE. Therefore, organizational learning capability will play a vital role in internal resistance mitigation, a prerequisite for successful IT intervention [30]. Rethinking organizational, legal implications in line with the need for a technologydriven intervention could facilitate BcT-based HIE implementation.

\subsubsection{Environmental Challenges}

Developing an integrated nationwide HIE requires collaboration among competitors. There are a few network barriers within the industry amongst competitors. A push from central authorities and a collaborative mindset among stakeholders is necessary for BcT intervention in the healthcare setting. BcT is a disruptive technology that requires advanced collaboration, social and user readiness, and the adoption behavior of competitors because a single health entity cannot establish the BcT for the entire HIE network. Missing goal alignment among participating entities is a common scenario since, currently, organizations are functioning individually. The concept of BcT allows the free flow of medical data among different healthcare entities, which are rivals from a business point of view.

Consequently, bringing all care delivery organizations under an umbrella and connection through BcT is complicated for the government and decision-makers. Many stakeholders prioritize organizational business incentives over-sharing and considering a global opportunity for peers. This silo poses a real struggle in an industrial setting [31]. Health entities are competitors. Therefore, data sharing can be seen as losing control over resources and providing others with a competitive advantage.

Stakeholders' trust and readiness need to be fostered further. Thus, government regulations and industrial policies should be redefined, as the current policies are not BcT friendly. Lack of trust is sure to hinder the diffusion and adoption of this technology [29]. For instance, according to the Malaysian Medical Council, guideline 2006 mentions that "A patient's medical record is the medical practitioner's property and the healthcare facility and services, who hold all rights associated with ownership" of public health entities. Also, regulation 44 (1), 2006, applicable for private health entities, states that "A patient's medical record is the property of a private healthcare facility or service." Therefore, the health entities cannot share the EMR data with patients, and the next of kin is also considered a third party. Hence, data sharing cannot take place until the restriction is lifted.

Furthermore, industrywide medical data and data exchanging mechanism has standard uncertainty [17]. Established relationships and power structures, which generate structural constraints, could harm the implementation of BcT. Dominant stakeholders can use an established power imbalance to compel others [23], [32]. As a result, decentralized governance of BcT remains controversial, as policymaking must not be formed by only a few participating health entities in a distributed ledger. Variation is specific entities' normative roots that may cause cultural bias, including sequential rigors, and hinder collaboration. Such institutional distance negatively affects the formation of shared values, goals, or activities [33]. A completely new barrier in the BcT system is that participants, especially network initiators, may be required to take on a new role as network orchestrators. As an initiator, one oversees handling network externalities by creating and sustaining a proper network. Misconception could lead to the adoption of BcT that is inappropriate for a particular entity. Because of the excitement around BcT, health entities may be tempted to choose it to be a part of the $\mathrm{BcT}$ revolution [23]. 


\subsubsection{Human/Individual-level Challenges}

A novel innovation can be a massive failure if the users refuse to use it. Therefore, scholars suggest that scholars assess users' perspectives whenever any electronic health (eHealth) innovation is introduced in the healthcare industry. Since BcT is a new technology, users may not be ready to adopt this technology for many reasons. For instance, resistance to change due to misunderstandings about the need for change or worry about failure due to the lack of personal innovativeness technology affinity may influence individuals' adoption intention. Concerns about the confidentiality of medical data may influence individuals' perception of trust and risk. Fear of losing professional autonomy and additional workload may cause resistance to change and adopt BcT for HIE among health professionals. The professionals may also need training in this regard.

There is a need for early-stage assessment for BcT in the Malaysian healthcare industry. Before 2006, two Malaysian hospitals used IBM mainframe computers for patient admission to medical record tracking, and the whole system was paperless. The report further mentioned that in 1985, a teaching hospital in Kuala Lumpur utilized the information management administration system. In 2004 the hospital began using an upgraded Health Information System (HIS) to achieve an integrated eHealth system. This evidence clearly shows that Malaysia is an early adaptor of digital technology innovation.

However, to date, the HIS implementation has not yet reached the desired level, which, on the contrary, indicates that despite being an early adaptor, the diffusion of eHealth innovation in the country remains very slow. Today, many of Malaysia's industries are still stuck in Industry 3.0 or even at Industry 2.0 level. Under IR 3.0, the minimum requirement was to achieve widespread use of EMR and EHR. In short, health technology adaptation in Malaysia remains below expectation and still struggling to reach the IR 3.0 level. Therefore, while introducing BcT-enabled medical data sharing to the Malaysian healthcare ecosystem, the preimplementation assessment is required because of the industry's sluggish adaptation nature and the nature of the BcT itself.

Disruptive technology requires changes in infrastructure, policy, and users' traditional way of working; therefore, it is essential to determine the factors that potentially influence users' adoption of the technology. BcT is a new technology; thus, the stakeholders' intention toward BcT adoption in the healthcare industry remains unknown. Users are the focal priority of any eHealth initiative. Any eHealth innovation will fail no matter how novel the tool or system is if the end-users refuse to adopt it.
Therefore, users' perceptions of the technology cannot be overlooked. A technology must gain users' acceptance for continuance in the future [34]. BcT has grabbed scholars' attention in the healthcare context since 2016 and is yet to be implemented to its fullest potential. BcT is a disruptive technology by nature and has the potential to improve patient safety and operational efficiency. Also, it could mitigate the risk of medical data breaches as it eliminates the need for third-party involvement in medical data management. However, despite its numerous benefits, the adoption of digital health technologies, such as telemedicine, EMR, EHR, Digital Health, and eHealth, remained low and is still lagging. Although other industries are rapidly adopting BcT , the BcT is still in an infancy stage in the healthcare industry.

\section{Guideline, Limitation and Future Research Direction}

Issues categorized under environmental factors require attention and combined effort from government, policymakers, and healthcare facilities management. The responsible authorities need to carefully develop and reform laws, medical practice guidelines, and policies at the national and organizational levels. Medical data exchange and related ownership regulations require revision. For instance, by law, the patients ought to be authorized to control their medical data, which currently belongs to Malaysia's healthcare facility and physicians. Furthermore, one of the main challenges is human or individuals' lack of readiness. Internal users such as health professionals and external users such as suppliers and patients, insurance providers' readiness assessment in the pre-implementation phase can help ease the individual-level barrier. Healthcare is a service-oriented and data-driven industry that serves the community to improve people's lives. Health professionals are the primary and internal users of health information technology. Therefore, the influence of prosocial behavior, self-determination, task technology-fit, trust, risk, technology characteristics and features, environment factors, organizational factors on BcT adoption needs empirical investigation. Technology adoption in the Malaysian healthcare industry has been an issue for the last three decades. Appropriate organizational change management enhances technology adoption difficulties.

However, the present study has a few limitations. During data search, the study utilized specific keywords; thus, not all relevant studies may appear. Similar analysis can be conducted in the future to provide an up-to-date taxonomy of challenges considering Malaysia or other developing countries. 


\section{Conclusion}

The future of blockchain is promising. Eventually, it will be possible to address the current issues utilizing BcT as an underlying mechanism for EHR. It can assist in ensuring the privacy, security, authenticity of information that is beneficial for the Malaysian healthcare industry. To the best of the researcher's knowledge, this study is the first to systematically unearth the challenges that may hinder BcT's implication in the Malaysian healthcare industry. The novelty of this study is the specific focus on developing countries like Malaysia in implementing blockchain technology for medical data management. This study is one of the first studies to focus exclusively on the possibilities, necessity, and challenges of the BcT in the Malaysian healthcare industry context. It contributes to the body of knowledge by closing the highlighted research gap. However, this study fills the literature gap as systematic research is absent from this phenomenon. This study's insight might provide the government, policymakers, and managers with a preliminary understanding of the new technology, allowing them to plan accordingly to overcome the challenges. The BcT implication could facilitate the paradigm shift in the healthcare industry by stabilizing the desired integration among stakeholders. It could turn the healthcare ecosystem toward patient-centric healthcare service delivery, thereby improving patient safety. The BcT in healthcare is still an underexplored area of interest. The successful implication of the BcT diffusion needs a push from the government, the healthcare industry, and stakeholders.

\section{Acknowledgements}

The Ministry of Higher Education funded this project under FRGS, Registration Proposal No: FRGS/1/2020/SS01/UTM/02/6 \& UTM.

\section{References}

[1]. Kelly, M. M., Coller, R. J., \& Hoonakker, P. L. (2018). Inpatient Portals for Hospitalized Patients and Caregivers: A Systematic Review. Journal of hospital medicine, 13(6), 405-412.

[2]. Pai, M.M.M., Ganiga, R., Pai, R.M. et al. (2021). Standard electronic health record (EHR) framework for Indian healthcare system. Health Serv Outcomes Res Method 21, 339-362 https://doi.org/10.1007/s10742-020-00238-0

[3]. Heart, T., Ben-Assuli, O., \& Shabtai, I. (2017). A review of PHR, EMR and EHR integration: A more personalized healthcare and public health policy. Health Policy and Technology, 6(1), 20-25. https://doi.org/10.1016/j.hlpt.2016.08.002
[4]. Zhang, X., \& Poslad, S. (2018, May). Blockchain support for flexible queries with granular access control to electronic medical records (EMR). In 2018 IEEE International conference on communications (ICC) (pp. 1-6). IEEE.

[5]. Leeming, G., Cunningham, J., \& Ainsworth, J. (2019). A ledger of me: personalizing healthcare using blockchain technology. Frontiers in medicine, 6, 171.

[6]. Enaizan, O., Eneizan, B., Almaaitah, M., AlRadaideh, A. T., \& Saleh, A. M. (2020). Effects of privacy and security on the acceptance and usage of EMR: the mediating role of trust on the basis of multiple perspectives. Informatics in Medicine Unlocked, 21, 100450.

[7]. Anjum, H. F., Rasid, S. Z. A., Khalid, H., Alam, M. M., Daud, S. M., Abas, H., ... \& Yusof, M. F. (2020). Mapping research trends of blockchain technology in healthcare. IEEE Access, 8, 174244-174254.

[8]. Shahnaz, A., Qamar, U., \& Khalid, A. (2019). Using blockchain for electronic health records. IEEE Access, 7, 147782-147795.

[9]. Sharma, M., \& Joshi, S. (2021). Barriers to blockchain adoption in health-care industry: an Indian perspective. Journal of Global Operations and Strategic Sourcing, 14(1), 134-169.

[10]. Angraal, S., Krumholz, H. M., \& Schulz, W. L. (2017). Blockchain technology: applications in health care. Circulation: Cardiovascular quality and outcomes, 10(9), e003800.

[11]. Tandon, A., Dhir, A., Islam, N., \& Mäntymäki, M. (2020). Blockchain in healthcare: A systematic literature review, synthesizing framework and future research agenda. Computers in Industry, 122, 103290.

[12]. Durneva, P., Cousins, K., \& Chen, M. (2020). The current state of research, challenges, and future research directions of blockchain technology in patient care: Systematic review. Journal of medical Internet research, 22(7), e18619.

[13]. Heston, T. F. (2017). A Case Study in Blockchain Healthcare Innovation. Authorea Preprints.

[14]. Kshetri, N. (2018). Blockchain and electronic healthcare records. Computer, 51(12), 59-63.

[15]. Yaqoob, S., Khan, M. M., Talib, R., Butt, A. D., Saleem, S., Arif, F., \& Nadeem, A. (2019). Use of blockchain in healthcare: A systematic literature review. Int. J. Adv. Comput. Sci. Appl, 10(5), 10.

[16]. Mokhtar, M. F. M. (2020). The Law and Challenges to Access Medical Record for Medical Negligence Claims in Malaysia. Jurnal Undang-undang dan Masyarakat, 26, 43-50.

[17]. Seh, A. H., Zarour, M., Alenezi, M., Sarkar, A. K., Agrawal, A., Kumar, R., \& Ahmad Khan, R. (2020, June). Healthcare data breaches: Insights and implications. In Healthcare (Vol. 8, No. 2, p. 133). Multidisciplinary Digital Publishing Institute.

[18]. Lim, S. Y., Fotsing, P. T., Almasri, A., Musa, O., Kiah, M. L. M., Ang, T. F., \& Ismail, R. (2018). Blockchain technology the identity management and authentication service disruptor: a survey. International Journal on Advanced Science, Engineering and Information Technology, 8(4-2), 1735-1745. 
[19]. Guo, Y., \& Liang, C. (2016). Blockchain application and outlook in the banking industry. Financial innovation, 2(1), 1-12.

[20]. Steinfield, C., Markus, M. L., \& Wigand, R. T. (2011). Through a glass clearly: standards, architecture, and process transparency in global supply chains. Journal of Management Information Systems, 28(2), 75-108. https://doi.org/10.2753/MIS0742-1222280204.

[21]. Meier, J., \& Sprague, R. H. (1991). The evolution of interorganizational systems. Journal of Information Technology, 6(3), 184-191. https://doi.org/10.1057/jit.1991.32

[22]. Chen, C., Loh, E. W., Kuo, K. N., \& Tam, K. W. (2020). The Times they Are a-Changin'-Healthcare 4.0 Is Coming!. Journal of medical systems, 44(2), 14. https://doi.org/10.1007/s10916-019-1513-0

[23]. Seebacher, S., \& Schüritz, R. (2017, May). Blockchain technology as an enabler of service systems: A structured literature review. In International Conference on Exploring Services Science (pp. 12-23). Springer, Cham.

[24]. Chen, H. S., Jarrell, J. T., Carpenter, K. A., Cohen, D. S., \& Huang, X. (2019). Blockchain in healthcare: a patient-centered model. Biomedical journal of scientific \& technical research, 20(3), 15017.

[25]. Lee, K., Lim, K., Jung, S. Y., Ji, H., Hong, K., Hwang, H., \& Lee, H. Y. (2020). Perspectives of patients, health care professionals, and developers toward blockchain-based health information exchange: qualitative study. Journal of medical Internet research, 22(11), e18582.

[26]. Abdulnabi, M., Al-Haiqi, A., Kiah, M. L. M., Zaidan, A. A., Zaidan, B. B., \& Hussain, M. (2017). A distributed framework for health information exchange using smartphone technologies. Journal of biomedical informatics, 69, 230-250. https://doi.org/10.1016/j.jbi.2017.04.013
[27]. Ismail, N. I., \& Abdullah, N. H. (2017). Malaysia Health Information Exchange: A systematic review. Business and Economic Horizons (BEH), 13(1232-2019-731), 706-721. https://doi.org/10.15208/beh.2017.47

[28]. Karame, G. O., Androulaki, E., Roeschlin, M., Gervais, A., \& Čapkun, S. (2015). Misbehavior in bitcoin: A study of double-spending and accountability. ACM Transactions on Information and System Security (TISSEC), 18(1), 1-32.

[29]. Taherdoost, H. (2019). Importance of technology acceptance assessment for successful implementation and development of new technologies. Global Journal of Engineering Sciences, 1(3).

[30]. Hsu, C., Lin, Y. T., \& Wang, T. (2015). A legitimacy challenge of a cross-cultural interorganizational information system. European Journal of Information Systems, 24(3), 278-294. https://doi.org/10.1057/ejis.2014.33

[31]. Bala, H., \& Venkatesh, V. (2007). Assimilation of interorganizational business process standards. Information systems research, 18(3), 340362. https://doi.org/10.1287/isre.1070.0134

[32]. Arfi, W. B., Nasr, I. B., Khvatova, T., \& Zaied, Y. B. (2021). Understanding acceptance of eHealthcare by IoT natives and IoT immigrants: An integrated model of UTAUT, perceived risk, and financial cost. Technological Forecasting and Social Change, 163, 120437.

[33]. Dong, M. C., Fang, Y., \& Straub, D. W. (2017). The impact of institutional distance on the joint performance of collaborating firms: The role of adaptive interorganizational systems. Information Systems Research, 28(2), 309-331. https://doi.org/10.1287/isre.2016.0675

[34]. Hyla, T., \& Pejaś, J. (2019). eHealth integrity model based on permissioned blockchain. Future Internet, 11(3), 76. 